JOIRNMI, OF MODERN OPTICS, 1987, VOL. 34, No. 9, 1169-1178

\title{
Approproximate photocounting statistics of shot-noise light with arbitrary spectrum
}

\author{
M. C. TEICH \\ Columbia Radiation Laboratory and Center for Telecommunications \\ Research, Department of Electrical Engineering, Columbia University, \\ New York, New York 10027, U.S.A.
}

and B. E. A. SALEH

Department of Electrical and Computer Engineering, University of Wisconsin, Madison, Wisconsin 53706, U.S.A.

(Received 6 January 1987)

\begin{abstract}
The photocounting statistics of shot-noise light have been studied extensively. Light exhibiting such statistical properties arises in photongeneration processes that involve a two-stage cascade of Poisson-based events, such as cathodoluminescence. Expressions for the photocounting distributions are complex because they depend on the overall mean of the distribution, the photodetector counting time, and the spectrum of the light. A simple twoparameter distribution, the Neyman Type-A (NTA), is shown to provide an excellent approximation to the photocounting statistics of shot-noise light with arbitrary spectral properties. The NTA distribution therefore plays the same role for shot-noise light that the negative-binomial distribution plays for chaotic light.
\end{abstract}

\section{Introduction}

The shot-noise-driven doubly stochastic Poisson point process (SNDP) $[1,2]$ and the Thomas point process (TPP) [3] are useful for describing sequences of events that arise from a cascade of two Poisson-based point processes, when the temporal effects of the cascading process are taken into account. These point processes have found use in a variety of fields, including optics [1-8], vision $[4,6,9-11]$, and other disciplines ranging from biological information transmission to astrophysics $[1,3,4,12-14]$.

The SNDP is formed from a two-stage Poisson cascade, in which each event of a primary Poisson point process produces a virtual inhomogeneous rate function, which in turn is the source of a secondary non-stationary Poisson point process. The secondary processes are superimposed to form the overall (stationary) point process. The SNDP is a doubly stochastic Poisson point process (DSPP); it is also a special case of the Neyman-Scott cluster process $[1,12]$. The primary events are excluded from the overall point process in the SNDP; the TPP is distinguished only by the inclusion of the primary events. It has been shown that the SNDP counting distribution reduces to the simple Neyman Type-A (NTA) $[1,4,15]$ when cascading occurs instantaneously; the TPP counting distribution similarly reduces to the Thomas (which is similar to the NTA) in the absence of time dynamics $[3,16]$.

In the context of optics, the theory has been used to characterize the photocounting statistics of shot-noise light. Light exhibiting such statistical 
characteristics arises when the photon-generation process involves a two-stage cascade of Poisson-based events. Examples include cathodoluminescence, optical fluorescence initiated by ionizing radiation, and $\mathrm{X}$-ray radiography. In processes such as these, both the impinging excitations (primary events) and the photon emissions (secondary events) are often endowed with Poisson statistics. The presence of Cerenkov photons can be accommodated by including the primary events [3].

Unfortunately, expressions for the photocounting distributions associated with shot-noise light are complex because they depend on the overall mean of the distribution $\langle n\rangle$, the counting time $T$ and a function $h(t)$. This function is an inhomogeneous rate that describes the time course of secondary-event production upon the arrival of a primary event, and it determines the spectrum of the light. The principal parameters that characterize $h(t)$ are the average number of secondary events per primary event $\alpha$ (the area of $h(t)$ ) and the characteristic secondary-event cluster time $\tau_{\mathrm{p}}$ (the width of $h(t)$ ).

The purpose of this paper is to investigate the validity of the Neyman Type-A distribution as an approximation for the photocounting statistics of shot-noise light with arbitrary spectrum. It is a simple distribution characterized by two parameters: its mean $\langle n\rangle$ and a multiplication parameter $a$. The choice of this particular distribution follows from theoretical studies demonstrating that the SNDP and TPP counting distributions approach the NTA in various limits [1,3]. Our principal interest is in the region $\beta=T / \tau_{\mathrm{p}} \approx 1$, where the specific form of $h(t)$ is likely to be important. The counting time $T$ is then of the order of the characteristic splay-out time of the secondary pulses $\left(\tau_{p}\right)$ so that the validity of the approximation must be determined numerically. The NTA approximation turns out to be excellent when $h(t)$ assumes two common forms (rectangular and exponential), for all regions of the parameters $\alpha$ and $\beta$. Our results suggest that the approximation is probably quite suitable for arbitrary $h(t), \alpha$ and $\beta$.

The approach we adopt is similar to that used by Bédard, Chang and Mandel [17] and by Perrina and his coworkers [18-21]. The former study dealt with the use of the negative-binomial distribution as an approximation for the photocounting statistics of chaotic light with arbitrary spectrum. The latter studies were concerned with the use of the non-central-negative-binomial (Laguerre) distribution as an approximation for the photocounting statistics of mixed coherent and chaotic light [22].

\section{The NTA counting distribution}

The NTA distributed random variable $n$, of mean $\langle n\rangle$ and multiplication parameter $a$ (average number of secondary events per primary event), has a momentgenerating function given by $[1,15]$

$$
Q_{n}(s) \equiv\langle\exp (-s n)\rangle=\exp \left\{\frac{\langle n\rangle}{a}[\exp [a(\exp (-s)-1)]-1]\right\}
$$

Its variance is

$$
\operatorname{Var}(n)=(1+a)\langle n\rangle
$$


The usual expression for the NTA counting distribution $p(n)$ takes the form of an infinite series [4], with a recurrence relation of the form [1]

$$
\left.\begin{array}{rl}
(n+1) p(n+1) & =\langle n\rangle \sum_{j=0}^{n} \exp (-a) \frac{a^{j}}{j !} p(n-j), \\
p(0) & =\exp \left[\frac{\langle n\rangle}{a}(\exp (-a)-1)\right] .
\end{array}\right\}
$$

When $a \rightarrow 0,(1)-(3)$ reduce to expressions for the Poisson distribution.

\section{Comparison of the NTA and SNDP counting distributions}

The NTA is considered a candidate for approximating the SNDP and TPP counting distributions because it is known from theoretical arguments that it approaches these complex counting distributions in important limits. For vanishingly small $\alpha$, or for vanishingly small $\beta$ and $\beta \alpha$, it has been shown that the SNDP counting distribution approaches the Poisson distribution for arbitrary $h(t)[1,2]$. This is because the secondary pulse clusters are then either very sparse $(\alpha \ll 1)$ or are cut apart by the small value of $T(\beta \ll 1)$. Since the NTA approaches the Poisson distribution for vanishingly small $a$, it certainly is a good approximation to the SNDP in this region. At the opposite extreme, when $\beta \gg 1$, the SNDP counting distribution also approaches the NTA for arbitrary $h(t)[1,2]$. This is because all of the secondary pulses are then captured in the long counting time $T$ since $T \gg \tau_{\mathrm{p}}$.

The comparison is effected by matching the NTA mean and variance (2) to the mean and variance of the SNDP counting distribution, which is given by

$$
\operatorname{Var}(n)=\left(1+\frac{\alpha}{\mathscr{M}}\right)\langle n\rangle .
$$

The quantity $\mathscr{M}$ is a degrees-of-freedom parameter that is determined by $h(t)$ and $T$. When $h(t)$ has the rectangular form

$$
h(t)=\frac{\alpha}{\tau_{\mathrm{p}}} \quad\left(0 \leqslant t \leqslant \tau_{\mathrm{p}}\right)
$$

the degrees-of-freedom parameter $\mathscr{U}(\mathrm{SNDP}$, rect) becomes [1]

$$
\mathscr{M}(\mathrm{SNDP}, \text { rect })= \begin{cases}\left(\beta-\frac{1}{3} \beta^{2}\right)^{-1}, & (\beta \leqslant 1), \\ \beta\left(\beta-\frac{1}{3}\right)^{-1}, & (\beta \geqslant 1) .\end{cases}
$$

The usefulness of the NTA as an approximation to the SNDP counting distribution with spectral properties determined by the rectangular form of $h(t)$ is illustrated graphically in figure 1 . Six representative SNDP counting distributions $p(n)$ are shown versus count number $n$ for various values of the SNDP multiplication parameter $\alpha$ and the capture ratio $\beta=T / \tau_{\mathrm{p}}$. These distributions were calculated from the recursion relations derived earlier [1]. The mean count $\langle n\rangle$ is 10 in all cases. Also shown in figure 1 are six NTA distributions whose mean and variance have been matched to those of the SNDP counting distributions by equating (2) and (4), and by using (6) to solve for $a$ in terms of $\alpha$ and $\beta$. In several panels of figure 1 , the two curves are so nearly identical that they look like a single curve.

In figures $1(a) \cdots(c), \alpha$ is chosen to be unity, whereas in figures $1(d)-(f), \alpha=10$; $\beta=0.05$ at the top panels, increasing to 5.0 at the bottom panels. It is apparent from 

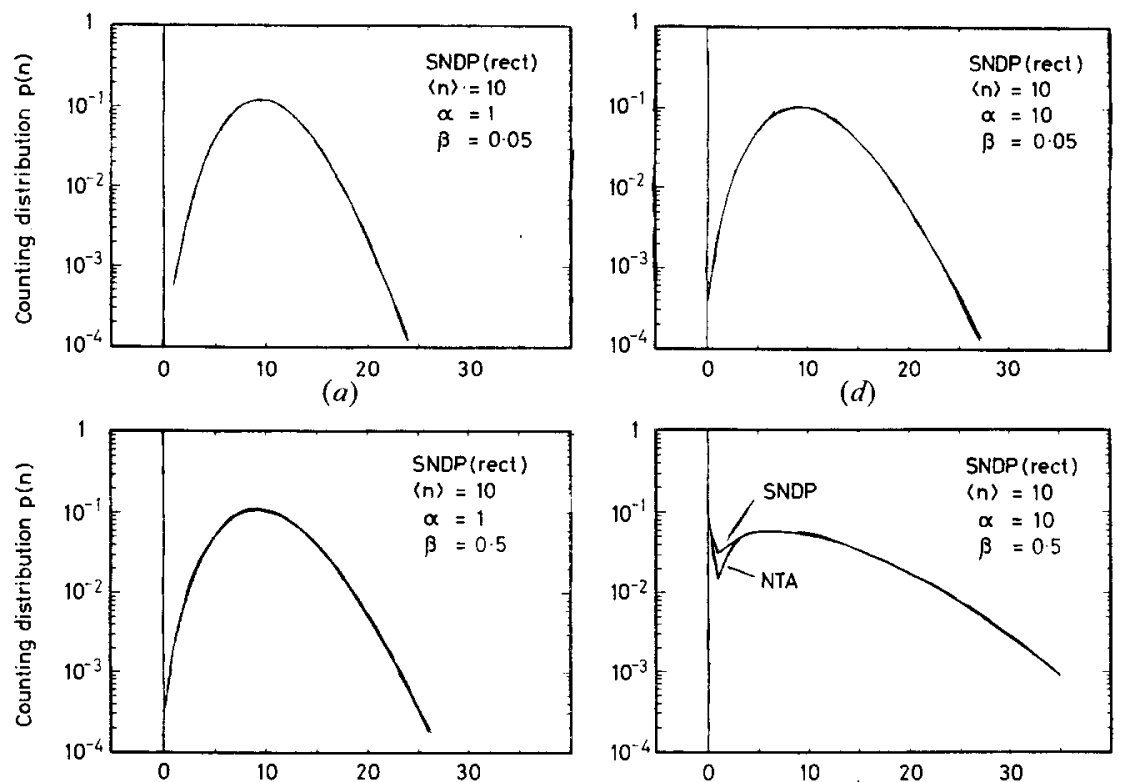

(b)

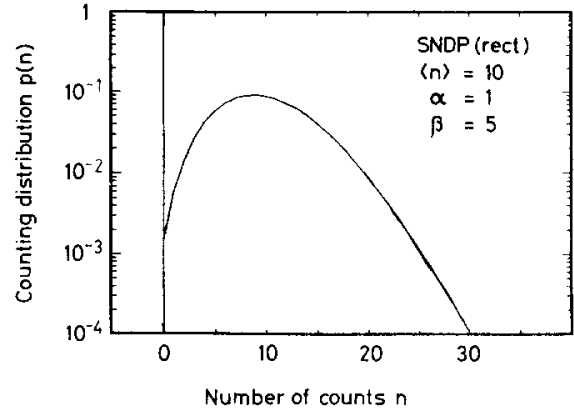

(c)

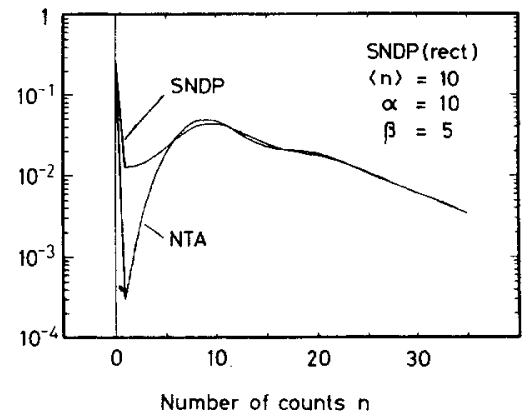

(f)

Figure 1. Six representative rectangular SNDP counting distributions compared with NTA distibutions of the same mean $(\langle n\rangle=10)$ and variance. The multiplication parameter $\alpha$ and the time parameter $\beta$ are indicated in each panel.

figure 1 that NTA and rectangular SNDP distributions of the same mean and variance are indistinguishable for $\alpha=1$ regardless of the value of $\beta$. The same is true for $\alpha=10$ and $\beta=0.05$, as in figure $1(d)$; this is expected because the SNDP distribution reduces to the NTA when $h(t)$ is rectangular in shape and the counting time is very short $(\beta \ll 1)[1]$. However, the NTA provides a less-than-perfect approximation for certain ranges of the count number $n$, when $\alpha=10$ and $\beta=0.5$ and 5.

Similar results are shown in figure 2 when $h(t)$ assumes the exponential form

$$
h(t)=\frac{2 \alpha}{\tau_{\mathrm{p}}} \exp \left(\frac{-2 t}{\tau_{\mathrm{p}}}\right) \quad(t \geqslant 0),
$$




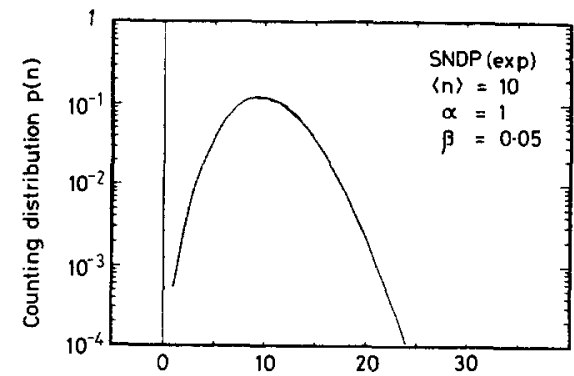

(a)

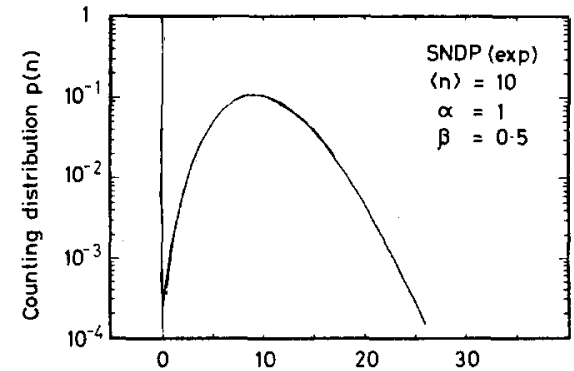

(b)

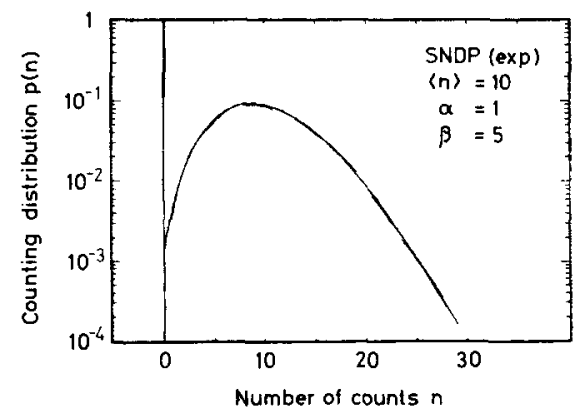

(c)

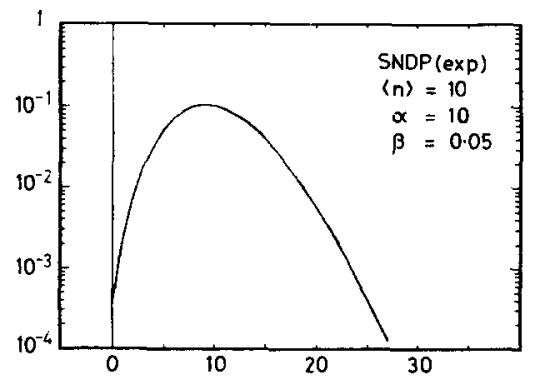

(d)

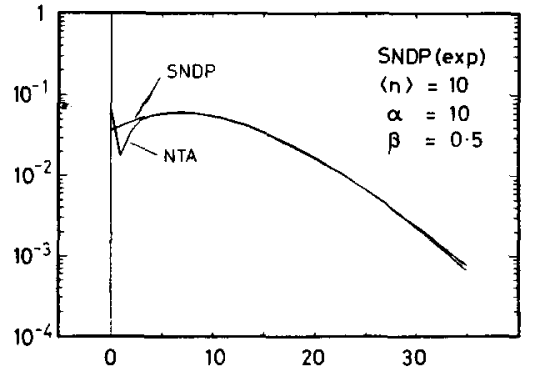

(e)

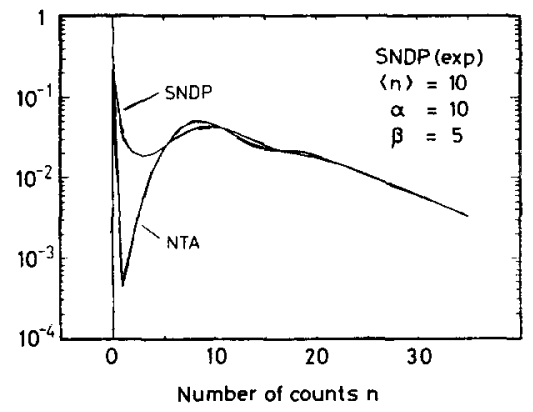

$(f)$

Figure 2. Six representative exponential SNDP counting distributions compared with NTA distributions of the same mean $(\langle n\rangle=10)$ and variance. The parameters $\alpha$ and $\beta$ are indicated.

in which case the exponential degrees-of-freedom parameter $\mathscr{M}$ (SNDP, exp) is given by [1]

$$
\mathscr{M}(\mathrm{SNDP}, \exp )=\frac{2 \beta}{\exp (-2 \beta)+2 \beta-1} .
$$

Again, various values of $\alpha$ and $\beta$ are represented in the individual panels of figure 2, and $\langle n\rangle=10$. The matching of the variance is again accomplished by equating (2) and (4), but now (8) is used to give $a$ in terms of $\alpha$ and $\beta$.

In figures $2(a)-(c), \alpha=1$, whereas in figures $2(d)-(f), \alpha=10 . \beta$ increases from 0.05 at the top panels to 5.0 at the bottom panels. Apparently the NTA and exponential SNDP distributions are also indistinguishable (within the graphical accuracy of the figures) when $\alpha=1$, independent of the value of $\beta$. In this case, however, there is a bit more disparity between the NTA and the exponential SNDP 
distribution when $\alpha=10$ and $\beta=0.05$ (figure $2(d)$ ); this is expected [1]. In fact, the NTA misses the mark by a bit for all cases in which $\alpha=10$. Nevertheless, a comparison of figures 1 and 2 demonstrates that the NTA fares just about as well in approximating rectangular and exponential SNDP counting distributions. The SNDP distributions appear to be smoothed versions of the NTA, in which the sharp dips of $p(n)$, at low values of $n$, are washed out. The tails of the distributions are approximately the same.

The goodness of the approximation can be quantitatively estimated. To accomplish this, we calculate the relative error $\delta(n)$ as a function of the count number $n$, where $\delta(n)$ (in \%) is defined as

$$
\delta(n)=100 \times \frac{|p(n, \mathrm{SNDP})-p(n, \mathrm{NTA})|}{p(n, \mathrm{SNDP})} .
$$

$\delta(n)$ is displayed in figures $3(a, b)$ for rectangular SNDP, and in $3(c, d)$ for exponential SNDP, counting distributions. The values of $\alpha$ and $\beta$ are indicated in the individual panels of the figure, and again $\langle n\rangle=10$. The error is oscillatory with the count number, indicating that the approximation is better for certain values of $n$. Overall, the relative error is often quite small, but it can become large for particular values of $n$. Expectations based on theoretical arguments are borne out. The NTA
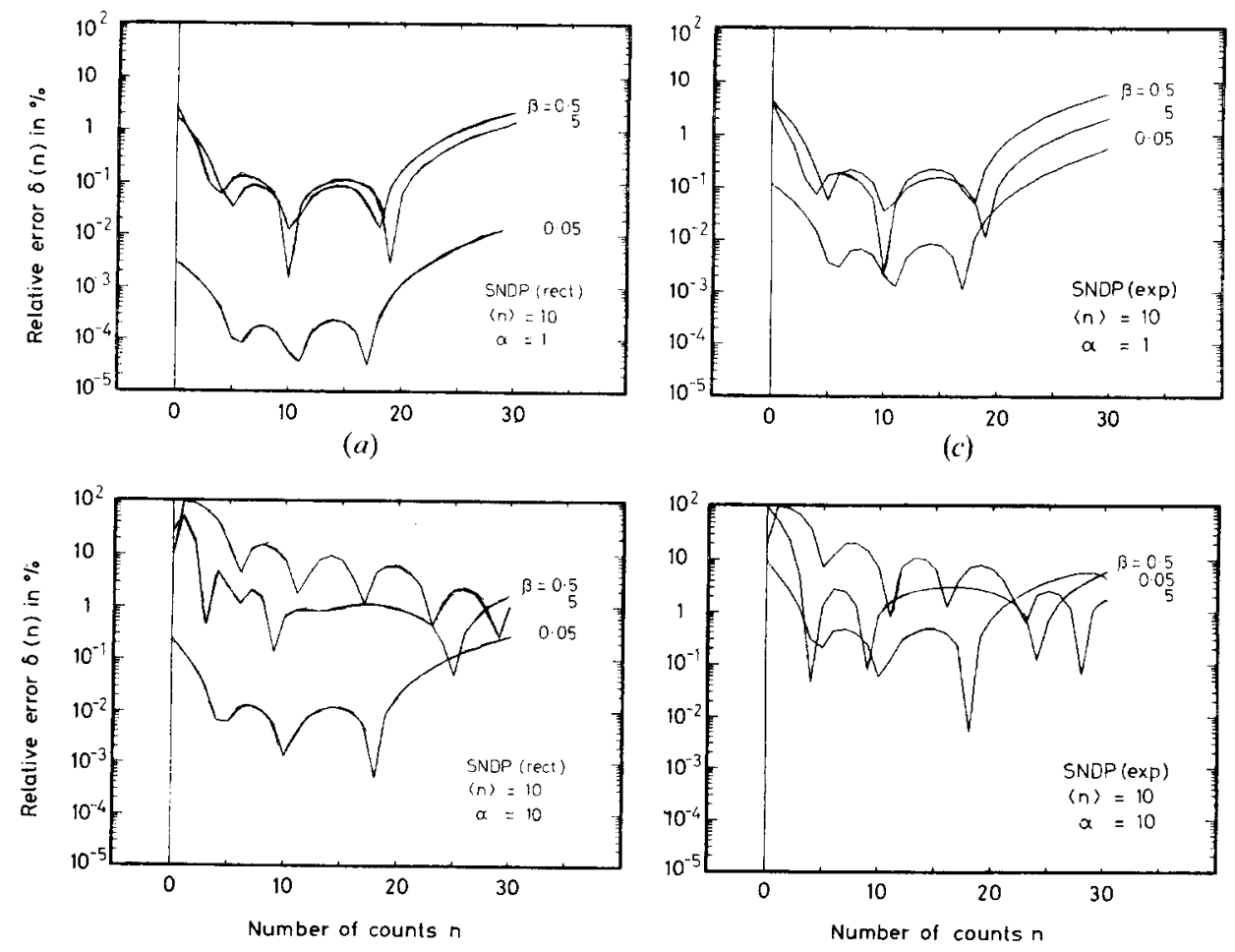

(b)

$(d)$

Figure 3. Relative error $\delta(n)$ (per cent) versus number of counts $n$ for rectangular and exponential SNDP counting distributions. These curves are obtained from the distributions in figures 1 and $2 .\langle n\rangle=10 ; \alpha$ and $\beta$ are indicated. 
provides a slightly better approximation for the rectangular SNDP distribution (i.e. the curves lie a bit lower) than for the exponential SNDP distribution. The approximation is always somewhat better for $\beta=0 \cdot 05$ than for higher values of $\beta$.

\section{Comparison of the NTA and Thomas-process counting distributions}

The NTA also approaches the TPP counting distribution in certain limits. The TPP counting distribution with arbitrary $h(t)$ becomes the Poisson distribution when $\alpha=0$, or when $\beta$ and $\beta \alpha$ become vanishingly small [3]. The first conditions represents a degenerate case in which there are no secondary events but Poisson primary events are carried forward, whereas the second condition represents a situation in which the secondary-pulse clusters are cut apart by the small value of $T$ $(\beta \ll 1)$. Since the NTA also approaches the Poisson when $a \rightarrow 0$, it clearly provides a good approximation to the TPP counting distribution in this limit. In the opposite case, when $\beta \gg 1$, all of the secondary events are captured in the long counting time $T$, along with the primary events, and the TPP counting distribution with arbitrary $h(t)$ approaches the Thomas distribution $[3,4,16]$, which in turn is similar to the NTA. Finally, in the limit $\beta \gg 1, \alpha \gg 1$, the TPP, SNDP and NTA counting distributions all approach the fixed multiplicative Poisson distribution [4].

The variance of the TPP counting distribution is [3]

$$
\operatorname{Var}(n)=\frac{1+3 \alpha / \mathscr{M}^{\prime}+\alpha^{2} \mid \mathscr{M}}{1+\alpha}\langle n\rangle,
$$

where $\mathscr{M}^{\prime}$ and $\mathscr{M}$ are Thomas degrees-of-freedom parameters that are determined by $h(t)$ and $T$. When $h(t)$ assumes the rectangular form represented in (5),

$$
\mathscr{M}(\mathrm{TPP}, \mathrm{rect})=\mathscr{M}(\mathrm{SNDP}, \mathrm{rect})
$$

as given by (6) and [3]

$$
\mathscr{M}^{\prime}(\mathrm{TPP}, \text { rect })= \begin{cases}3(1+\beta)^{-1} & (\beta \leqslant 1), \\ \beta\left(\beta-\frac{1}{3}\right)^{-1} & (\beta \geqslant 1) .\end{cases}
$$

The NTA and TPP counting distributions were compared in the same way as reported earlier. The means and variances of the NTA and the TPP distributions were matched by equating (2) and (10), and by using (6), (11) and (12) to provide an expression for $a$ in terms of $\alpha$ and $\beta$.

Six representative rectangular TPP counting distributions, along with six NTA distributions of the same mean and variance, are displayed in figure 4 . The TPP distributions were computed from the recursion relations obtained previously [3]. The mean $\langle n\rangle$ was again chosen to be 10 , and the same values of $\alpha$ and $\beta$ were used as in figure 1 for the SNDP. In four panels of figure 4 the NTA and TPP curves are so nearly identical that they look like a single curve. Comparison of figures 1 and 4 reveals a great deal of similarity.

Again, the goodness of the approximation may be quantitatively established by use of the relative error $\delta(n)$ expressed in (9), substituting $p(n$, TPP) for $p(n, \mathrm{SNDP})$. The result is displayed in figure 5. The values of $\langle n\rangle, \alpha$ and $\beta$ are the same as those used for the SNDP in figures $3(a, b)$. Again, the NTA approximation is somewhat better for $\beta=0.05$ than for higher values of $\beta$. A general comparison of figures 5 and 3 quickly confirms that the NTA provides as suitable an approximation for the rectangular TPP distribution as it does for the rectangular SNDP distribution. 

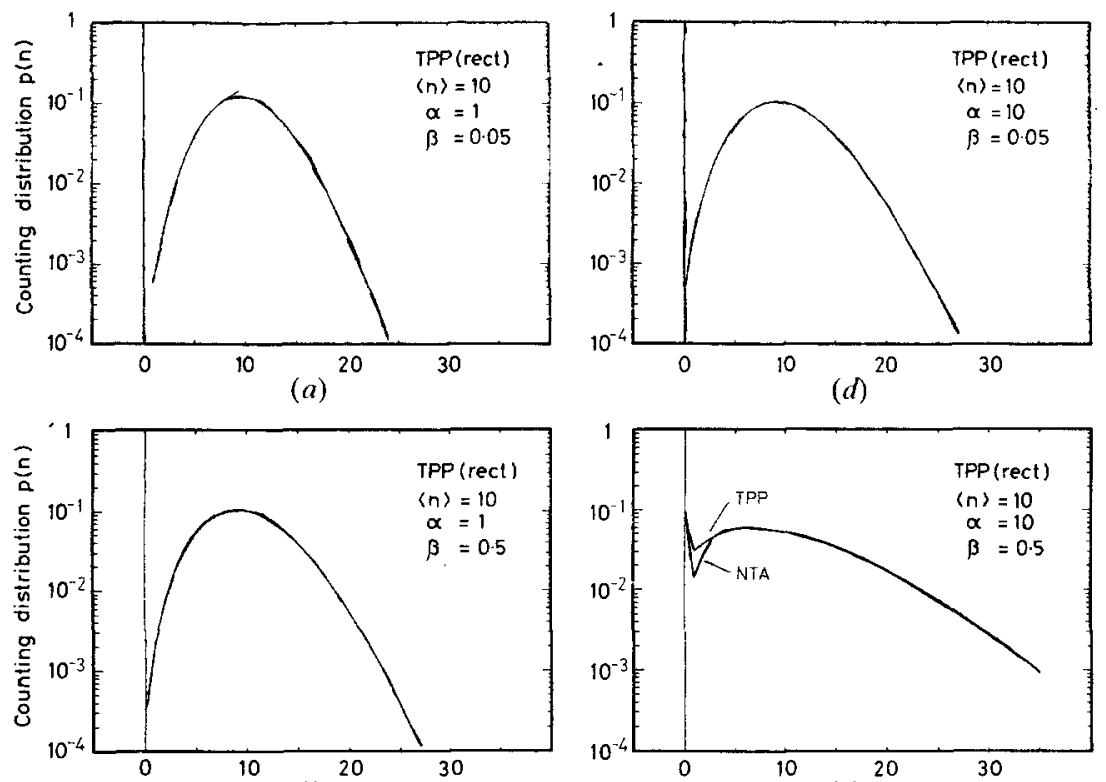

(b)

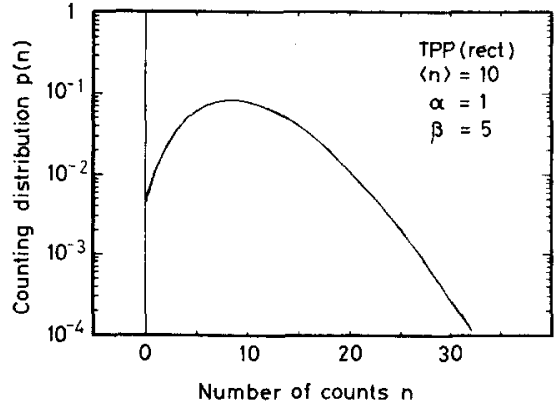

(c)

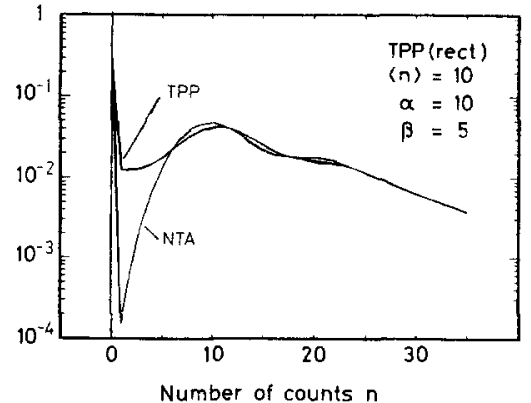

$(f)$

Figure 4. Six representative rectangular TPP counting distributions compared with NTA distributions of the same mean $(\langle n\rangle=10)$ and variance. The parameters $\alpha$ and $\beta$ are indicated.

We conclude that the NTA provides an excellent approximation for the SNDP and TPP counting distributions, with rectangular and exponential impulse response functions, when the mean and variance of the distributions are matched. The NTA distribution therefore plays the same role for shot-noise light that the negativebinomial distribution plays for chaotic light.

Finally, we note that counting distributions for cascaded Poisson processes with an arbitrary number of stages have recently been calculated $[23,24]$. As an extension of the work reported here, it would be of interest to examine the performance of the NTA distribution as an approximation to the cascaded-Poisson family of counting distributions. A similar approach may also be useful for approximating non-Poisson cascaded processes, such as those used for describing the generation of antibunched and sub-Poisson light [25]. Aside from the NTA, many two-stage simple timeindependent counting distributions have been developed over the years. 


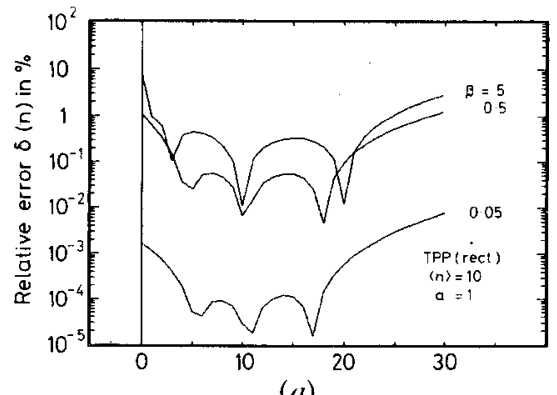

(a)

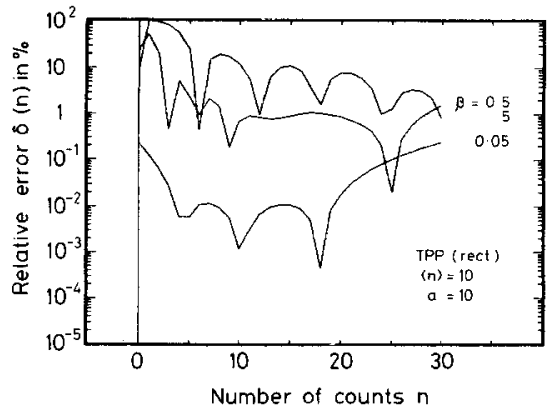

(b)

Figure 5. Relative error $\delta(n)$ (per cent) versus number of counts $n$ for rectangular 'IPP counting distributions. These curves are obtained from the distributions in figure 4 : $\langle n\rangle=10 ; x$ and $\beta$ are indicated.

\section{Acknowledgment}

We are grateful to Sein Lee and James Ritcey for computational assistance. This work was supported by the U.S. Joint Services Electronics Program and by the U.S. National Science Foundation.

\section{References}

[1] SAleh, B. E. A., and Teich, M. C., 1982, Proc. Inst. elect. electron. Engrs, 70, 229-245.

[2] Teich, M. C., and Saleh, B. E. A., 1981, Phys. Rev. A, 24, 1651-1654.

[3] Matsuo, K., Teich, M. C., and Saleh, B. E. A., 1983, Appl. Opt., 22, 1898-1909.

[4] Теich, M. C., 1981, Appl. Opt., 20, 2457-2467.

[5] Saleh, B. E. A., TavolaCci, J. T., and Teich, M. C., 1981, IEEE Jl quant. Electron., 17, 2341-2350.

[6] Saleh, B. E. A., and Teich, M. C., 1983, IEEE Trans. Inf. Theory, 29, 939-941.

[7] Saleh, B. E. A., Stoler, D., and Teich, M. C., 1983, Phys. Rev. A, 27, 360-374.

[8] Helstrom, C. W., and Rice, S. O., 1984, J. comput. Phys., 54, 289-324.

[9] Teich, M. C., and Saleh, B. E. A., 1981, J. opt. Soc. Am., 71, 771-776.

[10] Teich, M. C., Prucnal, P. R., Vannucci, G., Breton, M. E., and McGill, W. J., 1982, J. opt. Soc. Am., 72, 419-431.

[11] Saleh, B. E. A., and Teich, M. C., 1985, Biol. Cybernet., 52, 101-107.

[12] Neyman, J., and Scott, E. L., 1958, Jl R. statist. Soc. B, 20, 1-43.

[13] Neyman, J., and Puri, P. S., 1976, Proc. natl Acad. Sci. U.S.A., 73, 3360-3363.

[14] Lawrence, A. J., 1972, Stochastic Point Processes: Statistical Analysis, Theory, and Applications, edited by P. A. W. Lewis (New York: Wiley-Interscience), pp. 199-256.

[15] Neyman, J., 1939, Ann. math. statist., 10, 35-57.

[16] Thomas, M., 1949, Biometrika, 36, 18-25. 
[17] Bédard, G., Chang, J. C., and Mandel, L., 1967, Phys. Rev., 160, 1496-1500.

[18] Peřina, J., 1967, Phys. Lett., 24A, 333-334.

[19] Peřina, J., Peřinová, V., Lachs, G., and Braunerová, Z., 1973, Czech. J. Phys. B, 23, $1008-1013$.

[20] Peřina, J., 1984, Quantum Statistics of Linear and Nonlinear Optical Phenomena (Dordrecht: Reidel), Chap. 5, p. 148.

[21] Hradil, Z., and Peřina, J., 1986, J. opt. Soc. Am. A, 3, 1328-1333.

[22] Saleh, B. E. A., 1978, Photoelectron Statistics (Berlin: Springer).

[23] Matsuo, K., Saleh, B. E. A., and Teich, M. C., 1982, J. math. Phys., 23, 2353-2364.

[24] Matsuo, K., 'Teich, M. C., and Saleh, B. E. A., 1984, J. math. Phys., 25, 2174-2185.

[25] Teich, M. C., Saleh, B. E. A., and Peřina, J., 1984, J. opt. Soc. Am. B, 1, 366-389. 